\title{
Using Influence Nets in Financial Informatics: A Case Study of Pakistan
}

\author{
Sajjad Haider ${ }^{1}$, Muhammad Shafqat ${ }^{2}$ and Shabih Haider ${ }^{3}$ \\ ${ }^{1}$ Faculty of Computer Science, Institute of Business Administration, Karachi, Pakistan \\ 2 UNESCO, Islamabad, Pakistan* \\ ${ }^{3}$ Department of Economics and Finance, Institute of Business Administration, Karachi, Pakistan
}

The paper presents an application of Influence Nets (INs) in the field of financial informatics. Influence Nets have primarily been used in war games to model effects based operations but, as shown in this paper, they can prove to be equally useful in other domains requiring decision making under uncertain situations. The primary advantage of INs lies in their ability to acquire knowledge from subject matter experts in problem domains that rely heavily on experts' opinion. A sample case study from the fields of economics and finance is presented in this paper. The case study models the choices faced by a developing country to recover her economy which is going through a difficult phase due to global financial crisis, internal law and order situation and political instability.

Keywords: influence nets, Bayesian networks, AI in finance, causal maps, financial informatics

\section{Introduction}

Bayesian belief networks [1] have become the tool of choice for reasoning under uncertainty. BNs and their variants, such as Influence diagrams, Influence Nets and Bayesian Causal Maps, have been applied in many fields including fault diagnosis, information fusion, forensics, marketing, medical sciences and financial informatics, etc. [2-5]. Mathematically, a BN is a directed acyclic graph (DAG) where nodes in the graph represent random variables while arcs between pairs of nodes represent certain conditional independence assumptions. The DAG defines a factorization of the joint probability distribution of the modeled random variable. This joint distribution is obtained as a product of all conditional probabilities specified for each variable given its parent in the DAG. Formally,

$$
P\left(X_{1}, X_{2}, \ldots, X_{N}\right)=\prod_{i=1}^{N} P\left(X_{i} \mid p a\left(X_{i}\right)\right) .
$$

The main purpose of building a $\mathrm{BN}$ is to do probabilistic inference which involves computing the posterior probabilities of the variable(s) of interest after getting evidence about certain variables. The process is also referred to as belief updating. Several exact and approximate algorithms have been developed that exploit the structure of a BN to do belief updating in an efficient manner [6-10]. It must be stated, however, that, in general, the belief updating in a multiply-connected $\mathrm{BN}$ (where at least two nodes are connected through multiple paths) is NP-Hard [11].

When sufficient data is available, the structure and parameters of a $\mathrm{BN}$ can be learned from it. However, if the data is not available, then it becomes the job of a knowledge engineer, together with subject matter experts, to completely specify a BN. Most of the complex unprecedented situations belong to this category. The BN specification process involves building the structure of the network as well as filling the corresponding conditional probability tables. One of the major limitations of BNs is the acquisition of these conditional probabilities. They grow exponentially with the number of parents of a node, thus making it impossible for a subject matter expert to specify them.

\footnotetext{
${ }^{*}$ Muhammad Shafqat was studying at the Faculty of Computer Science, Institute of Business Administration, Karachi when the work was done.
} 
Several schemes have been suggested to overcome this limitation. The list includes NoisyOr [12], the CAST logic $[13,14]$ and some other extensions of Noisy-Or (including Generalized Noisy-Or [15] and Recursive Noisy-Or [16]). Such schemes ask for a non-exponential (primarily linear) number of parameters from a subject matter expert and then transform these parameters into conditional probabilities required for the full specification of a BN.

The special instance of BNs which employs the CAST logic for knowledge elicitation is referred to as Influence Nets (INs). The CAST logic based interface of INs allows a subject matter expert to model both positive and negative impacts of an event on other events, again with a limited number of parameters. Unlike NoisyOr, which asks an expert to specify the impact of an event on its child event, the CAST logic allows a subject matter expert to specify the impact of both the presence and absence of an event. In addition, instead of using a probabilistic scale of $[0,1]$, it allows an expert to use a non-probabilistic scale between -1 and 1 to specify impacts of the presence/absence of an event on its child event. This ability to capture both positive and negative impacts makes INs better equipped to model problem domains that rely heavily on experts' knowledge.

Two other techniques that also aim to capture positive and negative impacts are Bayesian Causal Maps (BCM) [17,18] and Qualitative Bayesian Networks (QBN) [19]. INs, however, offer a more expressive knowledge acquisition mechanism than its counterparts. Both BCM and QBN model a positive or negative impact of the presence of an event (i.e., the event being in the "True" state) on its child event, but do not model the impact of the absence of such event (i.e., the event being in the "False" state) on its child. There is no such limitation in the INs. Furthermore, QBN, as the name suggests, only allows the modeling of qualitative positive or negative strengths. INs, on the other hand, provide a mechanism to transform positive and negative impacts into conditional probability tables. Similarly, BCM employs qualitative positive and negative strengths during the early stages of model building phase when it uses causal maps to build the initial skeleton of the BCM. However, when it comes to the specification of conditional probabilities, BCM suf- fers from the same knowledge elicitation limitations as BNs because it requires an exponential number of conditional probability values.

INs and their extensions, Timed Influence Nets (TINs) [20] and Dynamic Influence Nets [21,22], have been extensively used in the field of effectsbased operations. They are used to model and evaluate alternative courses of action and their effectiveness to a mission's objectives [23-27]. Their scope, however, is not just limited to military and political analysis and they can prove to be equally useful in other domains requiring complex decision making. This paper presents an application of INs in the field of financial informatics. The paper shows how an IN can be used to model the choices faced by a developing country like Pakistan which, in recent years, is going through a serious economic crisis due to many factors including deteriorating law and order situation, political instability, high inflation and many global factors. It is also shown how techniques such as sensitivity analysis and sets of actions finder (SAF) algorithm can help a decision maker in understanding the impact of unprecedented events on the country's economy.

The rest of the paper is organized as follows. Section 2 provides an overview of Influence Nets, the CAST logic, sensitivity analysis and the SAF algorithm. Section 3 discusses the case study and explains how Influence Nets are used in financial informatics. Finally, Section 4 concludes the paper and provides the future research directions.

\section{Influence Nets}

Influence Nets are Directed Acyclic Graphs (DAGs) where nodes in the graph represent random variables, while the edges between pairs of variables represent causal relationships. The modeling of the causal relationships is accomplished by creating a series of cause and effect relationships between variables representing desired effect(s) and variables representing set of actionable events. Typically, the actionable events are drawn as root nodes (nodes without incoming edges), while the desired effect is modeled as a leaf node (node without outgoing edges). Influence Nets require a system modeler (or a subject matter expert) to specify the 


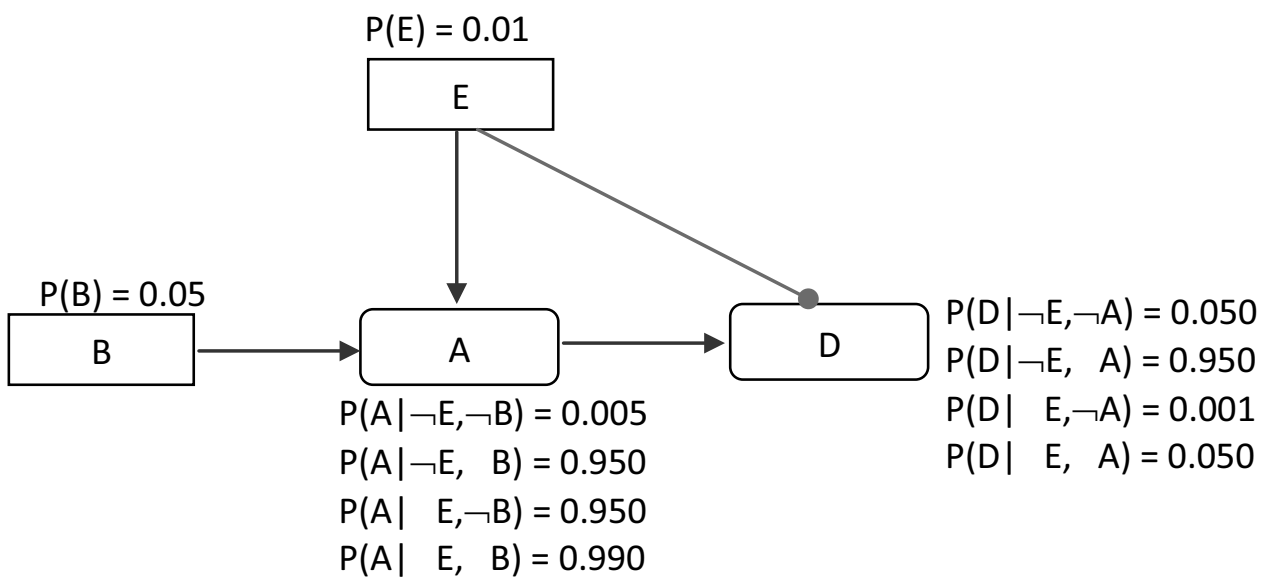

Figure 1. A sample influence net.

CAST logic parameters instead of the probabilities. The required probabilities are internally generated by the CAST logic algorithm with the help of user-defined parameters. The following items characterize an IN.

1. A set of random variables that makes up the nodes of an IN. All the variables in the IN have binary states.

2. A set of directed links that connect pairs of nodes.

3. Each link is associated with a pair of CAST logic parameters that show the causal strength of the link (usually denoted as h and g values).

4. Each non-root node has an associated CAST logic parameter (denoted as the baseline probability), while a prior probability is associated with each root node.

Figure 1 shows an example of an Influence Net. The directed edge with an arrowhead between two nodes shows the parent node promoting the chances of a child node being true, while the roundhead edge shows the parent node inhibiting the chances of a child node being true. The text associated with the non-root nodes represents the corresponding conditional probability values obtained from the CAST logic parameters (not shown in the figure) while the text associated with the root nodes represents the prior probabilities.

\subsection{CAST Logic}

The specification of a Bayesian Network requires an exponential number of parameters for model specification. As a model grows larger, this requirement presents a very big challenge to a system modeler. As an attempt to overcome this limitation, Chang et al. [14] developed a formalism called CAusal STrength (CAST) logic to elicit the large number of conditional probabilities from a small set of user-defined parameters. The logic has its roots in the Noisy-Or approach. The logic requires only a pair of parameter values for each dependency relationship between any two random variables. The values are converted into conditional probability tables and the resulting tables are used during the belief updating phase. Thus, Influence Nets could be regarded as a special instance of Bayesian Networks. A brief explanation of the CAST logic is provided below, with the help of an example shown in Figure 2. Readers interested in a detailed description of the CAST logic should refer to $[13,14]$.

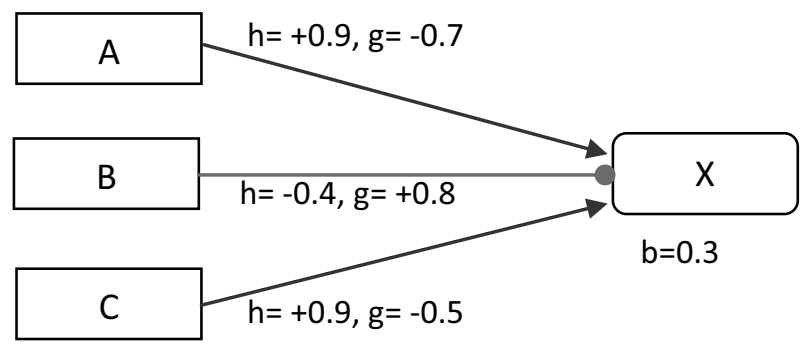

Figure 2. An influence network with CAST logic parameters. 
Figure 2 contains four nodes $\mathrm{A}, \mathrm{B}, \mathrm{C}$ and $\mathrm{X}$. On each arc, two causal strengths are specified. These numbers represent the probability that a specified state of a parent node will cause a certain state in the child node. Positive values on arcs are causal influences that cause a node to occur with some probability, while negative values are influences that cause the negation of a node to occur with some probability. For instance, the arc between $\mathrm{B}$ and $\mathrm{X}$ has values -0.4 and 0.8 . The first value, referred to as $\mathrm{h}$, states that if $\mathrm{B}$ is true, then this will cause $\mathrm{X}$ to be false with probability 0.4 , while the second value, referred to as $\mathrm{g}$, states that if $\mathrm{B}$ is false, then this will cause $\mathrm{X}$ to be true with probability 0.8 . Both $\mathrm{h}$ and $\mathrm{g}$ can take values in the interval $(-1,1)$. All non-root nodes are assigned a baseline probability, which is similar to the "leak" probability in the Noisy-Or approach. This probability is the user-assigned assessment that the event would occur independently of the modeled influences in a net.

There are four major steps in the CAST logic algorithm that converts the user-defined parameters into conditional probabilities:

a) Aggregate positive causal strengths

b) Aggregate negative causal strengths

c) Combine the positive and negative causal strengths, and

d) Derive conditional probabilities

In Figure 2, there are eight conditional probabilities that need to be computed to obtain the marginal probability of X. Mathematically, the marginal probability of $\mathrm{X}$ is computed as:

$$
\begin{aligned}
P(X)= & P(X \mid \neg A, \neg B, \neg C) P(\neg A, \neg B, \neg C) \\
& +P(X \mid \neg A, \neg B, C) P(\neg A, \neg B, C) \\
& +P(X \mid \neg A, B, \neg C) P(\neg A, B, \neg C) \\
& +P(X \mid \neg A, B, C) P(\neg A, B, C) \\
& +P(X \mid A, \neg B, \neg C) P(A, \neg B, \neg C) \\
& +P(X \mid A, \neg B, C) P(\neg A, \neg B, C) \\
& +P(X \mid A, B, \neg C) P(A, B, \neg C) \\
& +P(X \mid A, B, C) P(A, B, C)
\end{aligned}
$$

The four steps, described above, are used to calculate each of these eight conditional probabilities. For instance, to calculate the probability $P(X \mid A, B, \neg C)$, the $\mathrm{h}$ values on the arcs connecting $\mathrm{A}$ and $\mathrm{B}$ to $\mathrm{X}$ and the $\mathrm{g}$ value on the arc connecting $\mathrm{C}$ to $\mathrm{X}$ are considered. Hence, the set of causal strengths is $\{0.9,-0.4,-0.5\}$.

Aggregate the Positive Causal Strengths: In this step, the set of causal strengths with positive influence are combined. They are aggregated using the equation

$$
P I=1-\prod_{i}\left(1-C_{i}\right) \quad \forall C_{i}>0
$$

where $C_{i}$ is the corresponding $\mathrm{g}$ or $\mathrm{h}$ value having positive influence and PI is the combined positive causal strength. For our example

$$
P I=1-(1-0.9)=0.9
$$

Aggregate the Negative Causal Strengths: In this step, the causal strengths with negative values are combined. The equation used for aggregation is

$$
N I=1-\prod_{i}\left(1-\left|C_{i}\right|\right) \quad \forall C_{i}<0
$$

where $C_{i}$ is the corresponding $\mathrm{g}$ or h value having negative influence and NI is the combined negative causal strength. Using the above equation, the aggregate negative influence is found to be:

$$
N I=1-(1-0.4)(1-0.5)=0.7
$$

Combine Positive and Negative Causal Strengths: In this step, aggregated positive and negative influences are combined to obtain an overall net influence. The difference of these aggregated influences is taken. The overall influence is obtained by taking the ratio of this difference and the corresponding promoting or inhibiting influence. Mathematically,

If $P I \geq N I$

$$
A I=\frac{P I-N I}{1-N I}
$$

If $N I>P I$

$$
A I=\frac{N I-P I}{1-P I} .
$$

Thus, the overall influence for the current example is

$$
A I=(0.9-0.7) /(1-0.7)=0.66
$$

Derive Conditional Probabilities: In the final step, the overall influence is used to compute 
the conditional probability value of a child for the given combination of parents.

$$
\begin{gathered}
P(\text { child } \mid j \text {-th state of parent states })= \\
=\left\{\begin{array}{c}
\text { baseline }+(1-\text { baseline }) \cdot A I \\
\text { when } P I \geq N I \\
\text { baseline }- \text { baseline } \cdot A I \\
\text { when } P I<N I
\end{array}\right.
\end{gathered}
$$

Using the above equation, $P(X \mid A, B, \neg C)$ is obtained as:

$$
P(X \mid A, B, \neg C)=0.3+0.7 \cdot 0.66=.762
$$

The steps explained above are repeated for the remaining seven conditional probabilities in Equation 1. It should be noted that, if the experts had sufficient time and knowledge of the influences, then the conditional probability table for each node can be used instead of $\mathrm{g}$ and $\mathrm{h}$ values. Furthermore, after estimating the conditional probability table, if some entries do not satisfy the expert, then those entries can be modified and then used for computing the marginal probability of a node.

\subsection{Tools to Gauge Impact of Changes in INs}

Once an IN model is developed, it can be further refined and analyzed using sensitivity analysis and sets of actions finder (SAF) algorithm. The sensitivity of action analysis looks at how sensitive an effect is with respect to the actionable events when actions are considered one at a time [28]. The analysis requires linear number of searches, i.e., if there are $n$ actionable events, then $n$ iterations are required to perform the analysis. The algorithm is presented in Table 1 .

Another approach to gauge the impact of actionable events on the desired effect is the sets of actions finder (SAF) algorithm. The SAF algorithm $[28,29]$ is a heuristic approach to determine the sets of actions (and their configuration/state) that cause the probability of the desired effect to be above (below) a given probability threshold. The algorithm achieves this task in significantly less time than is required for an exhaustive examination of the actions' search space, which is exponential in terms of

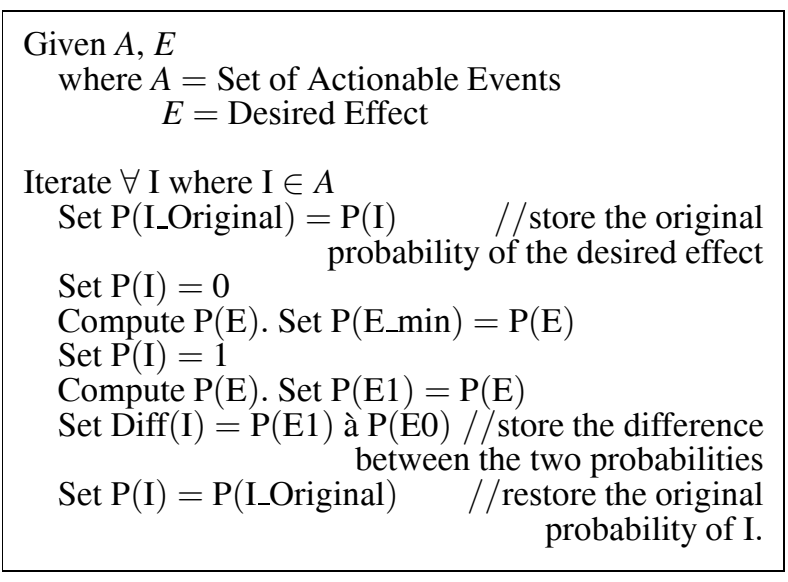

Table 1. Sensitivity of action analysis algorithm.

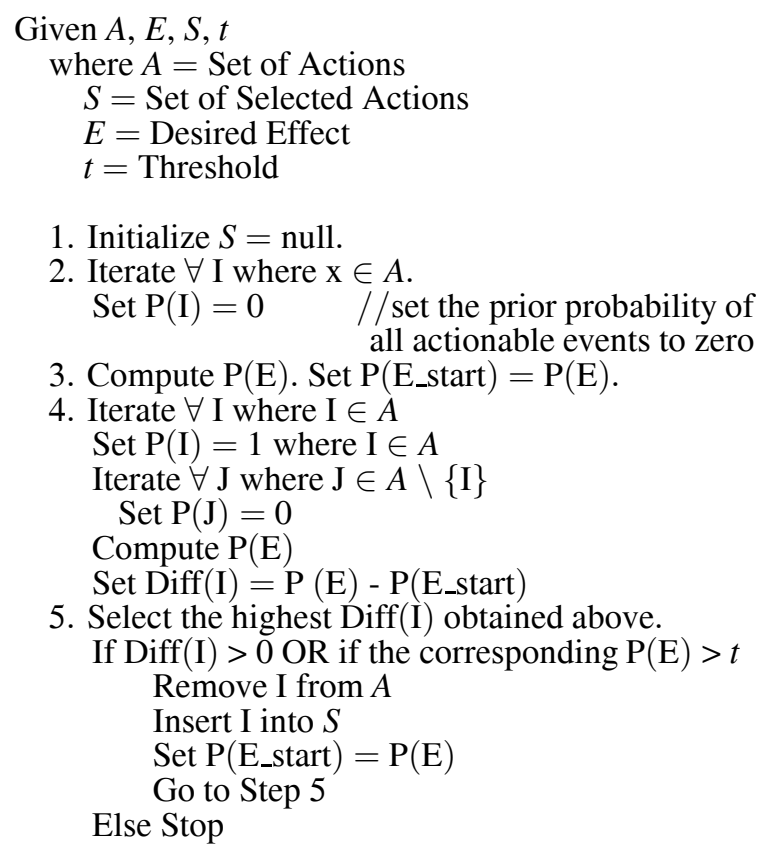

Table 2. The SAF algorithm.

the number of actions. The algorithm runs in polynomial time and uses a greedy approach to identify the best (or close-to-best) sets of actions. The algorithm is presented in Table 2.

\section{Case Study}

This section models and analyzes a financial decision making situation using Influence Nets. Unlike traditional modeling approaches that rely on empirical data, Influence Nets allow a knowledge engineer to capture experts' belief regarding the impact of unprecedented and unfold- 
ing events on the variable(s) of interests; and thus are better suited to model problem domains that rely heavily on subject matter experts' belief. These unprecedented events could include tsunami, earthquake, terrorist activities, food shortage, unusual hike in gold or oil price, etc.

The paper focuses on the economic/financial situation of Pakistan during the past few years. Being a growing economy, Pakistan has a huge potential in all areas, be it oil exploration, services sector, agriculture, industry, etc. Keeping this in view and also due to favorable political and financial situation between 2002 and 2007, Pakistan attracted a lot of foreign investment and saw a huge injection of funds from domestic investors. As a result, Karachi Stock Exchange, the biggest stock market of Pakistan, boomed from 1200 market index to 16000 market index. Starting from the first quarter of 2007 , however, this upward trend started moving in the opposite direction. The primary factors were global recession, deteriorating law and order situation, increase in terrorism, and political instability. These factors contributed to the stoppage of foreign investment and flight of capital. By 2008, the crisis reached its maximum and there was a danger that Pakistan could default as a country.

The Influence Net discussed in the sequel aims to structure the external/internal factors that impact, either positively or negatively, the state of Pakistani economy. The goal of this model building exercise is: (a) to integrate discrete economic indicators, (b) to illustrate the cause and effect relationships that exist among them, and (c) to present the use of this Influence Nets based model as a decision support tool in structuring the problem and making the right decision. Influence Net in this context coalesce the impact of individual variables onto subsequent nodes, thus ultimately building consequential outcome for the overall economy. In addition, by applying sensitivity analysis and the SAF algorithm, a knowledge engineer/decision maker can determine variables that have the greatest impact on the economy and in turn could aim to manipulate these variables to achieve the desired effect. It must be clearly stated that the focus of this paper is not to completely and accurately model all the relevant issues, but to highlight the advantages of IN based modeling technique using a reasonably valid (though hypothetical at times) picture of the selected scenario. In addition to sensitivity analysis and the SAF algorithm, the built IN model is also analyzed by having two different sets of prior probabilities for its root nodes (depicting actionable/observable events). These sets of prior probabilities reflect the chances of occurrence of the corresponding events in 2007/2008 and 2010 periods. Few of the actionable/observable events that impact the overall economy through chains of cause and effect relationships are described below.

External and Internal debt: Pakistan's economy has been mired with growing external and internal debt that aggravated into crisis-likesituation after the global financial crisis. Rise in general imports, flight of capital, widening trade and budget deficits, foreign debt servicing along with huge unsustainable subsidies in oil sector became the trigger for Pakistan's economic collapse. The government though gradually began passing on this pressure to people, but still it was not enough. Resultantly, all the gains of relieving economy from debt since 2002 reversed by 2008. Pakistan is still in the process of receiving foreign funding just to recover its economy, but things would get worse when the additional debt servicing starts of the newly "earned" debt.

IMF/WB-FOP Assistance: As discussed earlier that by early 2008, Pakistan came closer to getting default. It was ultimately offered loan (with strict conditions) by IMF, World Bank and Friends of Pakistan (FOP) consortium. The flow of assistance money from these sources helped it in boosting its Foreign Exchange reserves and stabilizing exchange rate.

Interest Rates: The central bank stabilized the market interest rates to contain inflationary pressure. The new policy of containing the consumption spree was in contrast to the expansionary policy of the previous seven years (till 2007) which was meant to demand pressure on import bills. Such mechanism helped in stabilizing exchange rate and Foreign Exchange reserves.

Commodity and Oil prices: There had been a sharp 'extraordinary' spike in oil and commodity prices in 2008 that virtually made economies collapse. Even though the prices fell after an abrupt spike, but the damage done in that relatively small period had a lasting impact on the economic gains of the past years. 
Remittances: Remittances by expats to Pakistan rose substantially since the start of global financial crisis. It is primarily triggered by massive layoffs by foreign companies that prompted capital to flow into home country. Such foreign flow of money helped in stopping the complete devaluation of Pakistan's currency which was triggered by the flight of capital.

Debt Servicing: Pakistan's substantial revenue goes to national debt servicing that has been on the rise for many years. Debt rescheduling and additional loans taken over the past decade are getting matured along with additional short terms debt. Such measures have a very negative impact on the foreign reserves and the exchange rate.

Tight Monetary Policy and Increased Taxes: In an attempt to reduce country's hunger for ever increasing imports and to encourage savings, the government pursued constricting monetary policy. Taxes had also been increased to close growing void between government's collection and spending.
Inflation and Cost of Production: Both factors were on high as a result of increasing demand and supply gap which was sparked by heightened oil prices that also increased electricity and transportation costs. These two, being at the core of any production, further increased every day commodity prices, thus making them expensive for people to afford.

Political instability and security: The repercussion of being a front line state in the global war on terror and having a fragile democratic political setup not only scared away prospective foreign investment, but also did not help in holding previous investments. This severely affected country's GDP growth.

The variables listed above are drawn as root nodes in the Influence Net. They impact (either positively or negatively) other intermediate events and ultimately all chains of cause and effect converge to a single desired effect "Improvement in Pakistani Economy". The complete model is shown in Figure 3. There are 17 root nodes which depict actionable (or potentially observable) variables. These root nodes impact 12 intermediate variables (not directly

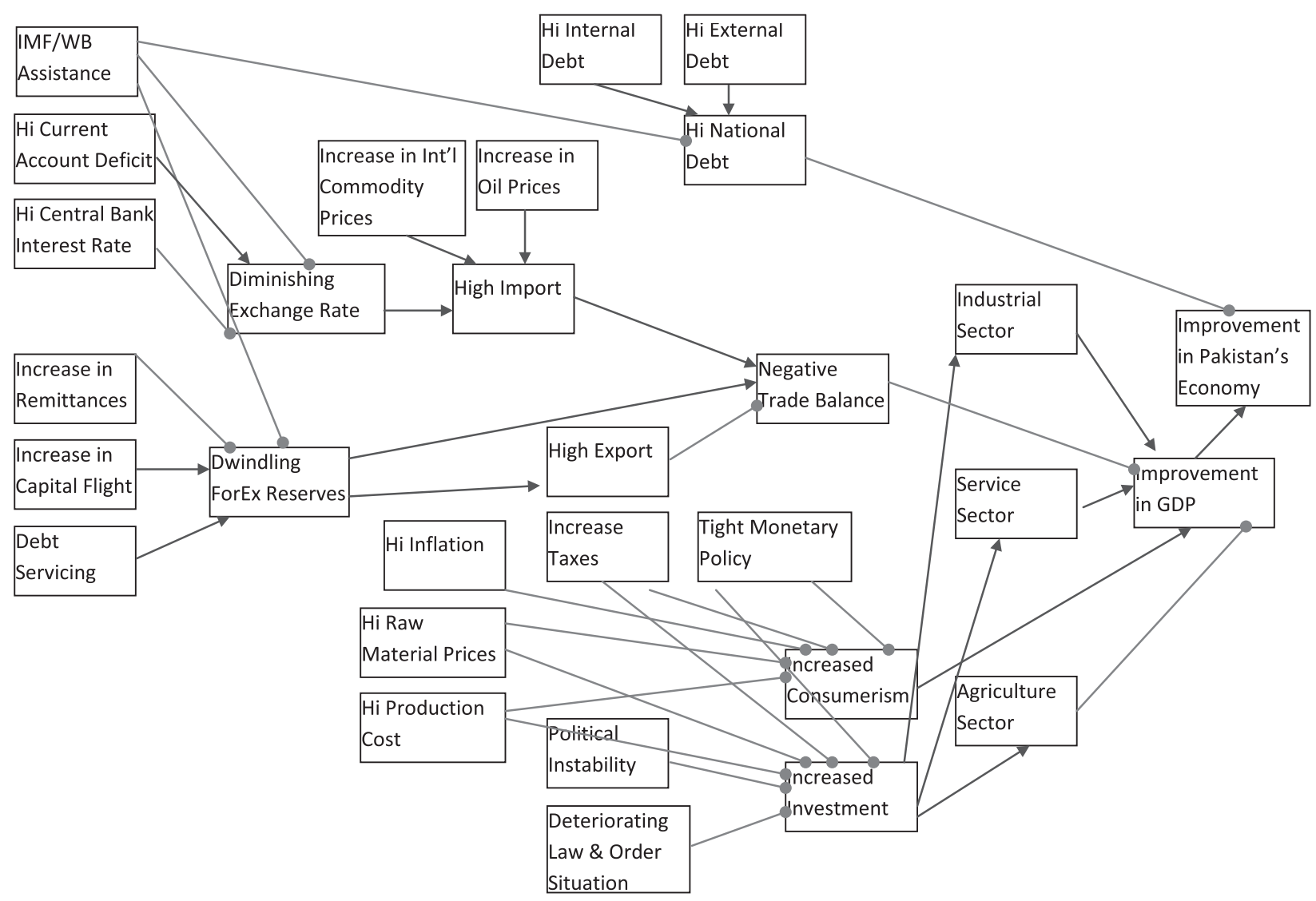

Figure 3. Influence net model of Pakistani economy. 
observables) and finally all of them directly or indirectly have an impact on the overall state of the Pakistani economy. The strength of positive or negative impact of the presence and absence of one variable on the other is quantified through the CAST logic. For instance, the impact of ' $\mathrm{Hi}$ Cntrl Bank Interest Rate' is much slower to surface on country's 'exchange rate' than the effect of 'WB or IMF assistance' that rescues depleting rates more briskly.

Once the influence net is completely built, its application lies in gauging the impact of actionable events by varying probabilities. In this study, two different sets of prior probabilities are considered. These sets of prior probabilities model the chances of occurrence of certain observable variables (root nodes) in 2007/2008 and 2010 periods. For instance, Pakistan has historically been relying heavily on assistance from World Bank and IMF that deemed necessary for bailing its ailing economy from doldrums. In 2007/2008, however, the economic conditions deteriorated when its plea for financial assistance was turned down and chances were not very bright of getting further aid. The situation is depicted with a very low prior probability (0.1) for the event "IMF/WB FOP Assistance". The situation, however, changed once the democratically elected government came into power, and since then, Pakistan has been receiving constant stream of financial assistance that greatly improved its deteriorating economic health. Thus, in 2010, the prior probability of "IMF/WB FOP Assistance" is quite high $(0.9)$. The probability is not set to 1 as there is always some element of uncertainty regarding the next installment if Pakistan fails to follow up on her promise to implement certain taxes and to reduce subsidies. Similarly, in 2007, international commodity prices reached a new height and broke all the previous records. Same was the case with oil prices which peaked to all time high. This causes the prior probabilities of these events to be very high $(0.9)$ in $2007 / 2008$. The situation is not so extreme in 2010 and prices have lowered down considerably from their peak values. This results in a lower prior probability $(0.3 / 0.4)$ for both variables.

Furthermore, due to emergency declaration in Pakistan which resulted in ban on judiciary and electronic media and, later on, due to the assassination of one of the most popular political figures, Pakistan faced an extremely high political instability in 2007/2008. In addition, the law and order situation was not very good due to the fallout of global war against terror, which resulted in many terrorist activities within the country. Thus, the corresponding events in the Influence Net, Political Instability and Deteriorating Law and Order Situation have a very high prior probability $(0.9 / 0.85)$. The situation in 2010 is slightly better as, despite many hiccups, the democratically elected government has been able to survive for more than 2 years. In addition, the army led operation against terrorists' hideout has marginally improved the law and order situation within the country. This results in slightly lower prior probabilities for both of these events in $2010(0.3 / 0.6)$. Table 3 lists all those factors which have significantly different prior probabilities during 2007/2008 and

\begin{tabular}{|l||c|c|}
\hline Variables & $\mathbf{2 0 0 7 / 2 0 0 8}$ & $\mathbf{2 0 1 0}$ \\
\hline \hline IMF/WB FOP Assistance & 0.1 & 0.9 \\
\hline Hi External Debt & 0.9 & 0.4 \\
\hline Incr Capital Flight & 0.9 & 0.5 \\
\hline Incr Intl Commodity Price & 0.9 & 0.4 \\
\hline Incr Oil Price & 0.9 & 0.3 \\
\hline Political Instability & 0.9 & 0.3 \\
\hline Deter. Law \& Order situation & 0.85 & 0.6 \\
\hline Hi Inflation & 0.8 & 0.4 \\
\hline \hline Improvement in Pakistani Economy & $\mathbf{0 . 0 8}$ & $\mathbf{0 . 1 7}$ \\
\hline
\end{tabular}

Table 3. Different sets of prior probabilities for $2007 / 2008$ and 2010 periods. 
2010 periods. Many events such as "Hi Internal Debt", "Tight Monetary Control", "Increase in Taxes", etc are not listed as their prior probabilities have not changed significantly from $2007 / 2008$ to 2010 . The table also lists the corresponding marginal probability of the desired effect "Improvement in Pakistani Economy". It can be seen that during the $2007 / 2008$ period, the chances of improvement in Pakistani economy were only $8 \%$. This number, however, has reached $17 \%$ in 2010, primarily due to financial assistance from IMF, World Bank and Friends of Pakistan consortium, running the country by a democratically elected government, and a substantial decrease in international commodity and oil prices. The value is still not very high as the country is going through a serious law and order situation and an extreme energy crisis which is resulting in lack of foreign/local investment and flight of capital.

Another way to analyze the impact of certain variables is through sensitivity analysis. As briefly discussed in Section 2.2, sensitivity analy- sis selects each node from the set of actionable nodes in an iterative manner. Its probability is first set to zero and then to one, while keeping the probabilities of other actionable events to their initial prior values. The sensitivity of the effect with respect to the selected action is determined by computing the probabilities of the desired effect when the action's probability is set to zero and one, respectively. At the end of this iterative process, the events which cause the highest difference in the likelihood of the occurrence of the desired effect are considered for further analysis. When sensitivity analysis is run on the Influence Net under consideration, the following five variables are identified as having the most significant individual impact on the state of Pakistani economy.

Hi Production Cost, Tight Monetary Policy, Deteriorating Law \& Order Situation, Hi External Debt, Hi Raw Material Prices

The sensitivity analysis is good to analyze the impact of individual events, but its outcome cannot be generalized to estimate collective impact

\begin{tabular}{|l||c|c|c|c|}
\hline \multicolumn{1}{|l||}{ Actionable Events } & \multicolumn{4}{|c|}{ State of Actionable Variables } \\
\hline & Set 1 & Set 2 & Set 3 & Set 4 \\
\hline \hline IMF/WB FOP Assistance & T & T & T & T \\
\hline Hi Current Acct Deficit & F & F & F & F \\
\hline Hi Cntrl Bank Interest Rate & T & T & T & T \\
\hline Hi Internal Debt & F & F & F & F \\
\hline Hi External Debt & F & F & F & F \\
\hline Incr. in Intl Commodity Price & F & F & F & F \\
\hline Incr. in Oil Price & F & F & F & F \\
\hline Incr. in Remittance & T & T & T & T \\
\hline Incr. in Capital Flight & F & F & F & F \\
\hline Debt Servicing & F & T & F & T \\
\hline Tight Monetary Policy & F & F & F & F \\
\hline Incr. in Taxes & F & F & F & F \\
\hline Hi Inflation & F & F & T & T \\
\hline Hi Raw Material Price & F & F & F & F \\
\hline Hi Production Cost & F & F & F & F \\
\hline Political Instability & F & F & F & F \\
\hline Deteriorating Law \& Order Situation & F & F & F & F \\
\hline \hline Improvement in Pakistani Economy & $\mathbf{0 . 7 9 9}$ & $\mathbf{0 . 7 9 8}$ & $\mathbf{0 . 7 9 5}$ & $\mathbf{0 . 7 9 3}$ \\
\hline
\end{tabular}

Table 4. Results of the SAF algorithm. 
due to non-synergistic and inhibitive impact of different variables, when taken together. Thus an actionable event, when considered alone, might have a positive impact on the desired effect, but the same variable might impact negatively when taken in synchronization with other variables. The collective impact of a set of actionable events is analyzed through the SAF algorithm, as briefly explained in Section 2.2. Primarily designed to support Influence Nets based analysis in effects based operations, the algorithm provides a mechanism to identify the best configuration(s) of actionable events that maximize the chances of achieving the desired effect. It starts with a single actionable event which, when considered individually, causes the highest increase (for a maximization problem) in the probability of the desired effect being true. This is followed by the selection of a second action from the remaining set of actions that together with the first action cause the highest increase in the probability of the desired effect. Other actions are added iteratively in a similar manner. The process stops at a point where (i) the inclusion of an action causes the probability of the objective node to decrease and to fall below a given probability threshold or (ii) there are no more actions to add. Once alternative sets of actions are obtained, they can be grouped together to form more general sets of actions.

When the SAF algorithm is run on Influence Net of Pakistani economy, it suggests 4 configurations of the set of actionable events that causes up to $80 \%$ chance of improvement in Pakistani economy. The actionable events and their configurations are listed in Table 4. A cursory look at these sets suggests that the configurations also match with the intuition one could develop by analyzing the model. All the actionable events have identical configurations (either true or false) except two events: "Debt Servicing" and "Hi Inflation" which have both true and false values. "IMF/WB and FOP Assistance" is true in all the solutions. Same is true for "Hi Remittance" and "Hi Cntrl Bank Interest Rate". Similarly variables like "Political Instability", "Deteriorating Law and Order Situation", "Hi External Debt", "Hi Intrl Commodity Price", etc are false in all the solutions. As discussed above and explained in .[28], these 4 solutions can be further generalized to form an aggregated solution.

\section{Conclusions}

The paper presents an application of Influence Nets in financial informatics. Influence Nets have been extensively used in modeling effects based operations, but, as shown in this paper, they can be equally useful in other domains that rely extensively on subjective knowledge. The primary advantage of Influence Nets lies in their capability to model both positive and negative impact of the presence/absence of an event on other events and that too with very limited number of parameters. They are also good for modeling unprecedented events which may not be adequately handled by empirical data based traditional approaches. Together with sensitivity analysis and the sets of actions finder algorithm, they can aid a decision maker/knowledge engineer in identifying the best sets of actions (and their corresponding state) which could maximize the chances of achieving the desired effect. The selected case study: (a) modeled the situation faced by a developing country like Pakistan as its economy suffered from global recession, political instability, deteriorating law and order situation during the past few years, and (b) suggested ways to overcome the burgeoning economic crisis. As mentioned earlier, the purpose of this study was not to accurately model all the economic factors, but to highlight the importance of Influence Nets based modeling technique. Nevertheless, the analysis and results presented in this paper are quite intuitive and the approach can easily be applied/generalized to other countries and/or situations with an enhanced set of variables.

\section{Acknowledgment}

The authors would like to thank the Editor and anonymous reviewers for their valuable comments and feedback, which were helpful in improving the paper.

\section{References}

[1] J. PEARL, Probabilistic Reasoning in Intelligent Systems: Networks of Plausible Inference, Morgan Kaufmann, 1988. 
[2] A. MitTal, Bayesian Network Technologies: Applications and Graphical Models, IGI Publishing, 2007.

[3] O. POURRET, P. NAĎM AND B. MARCOT, Bayesian Networks: A Practical Guide to Applications, Wiley, 2008.

[4] F. Taroni, C. Aitken, P. Garbolino And A. BieDERMANN, Bayesian Networks and Probabilistic Inference in Forensic Science, Wiley, 2006.

[5] R. E. NeAPOlitan AND X. Jiang, Probabilistic Methods for Financial and Marketing Informatics, Morgan Kaufmann, 2007.

[6] J. E. CANO, L. D. HERnÁNDEZ AND S. MORAL, Importance sampling algorithms for the propagation of probabilities in belief networks, Int. J. Approx. Reasoning, Vol. 15, 1996, pp. 77-92.

[7] J. CHENG AND M. J. DRUZDZEL, AIS-BN: An Adaptive Importance Sampling Algorithm for Evidential Reasoning in Large Bayesian Networks, Journal of Artificial Intelligence Research, Vol. 13, 2000, pp. 13-155.

[8] R. DECHTER, Bucket elimination: a unifying framework for reasoning, Artif. Intell., Vol. 113, 1999 , pp. 41-85.

[9] S. L. LAURITZEN AND D. J. SPIEGELHALTER, Local computations with probabilities on graphical structures and their application to expert systems, Readings in uncertain reasoning, Morgan Kaufmann Publishers Inc., 1990, pp. 415-448.

[10] D. Poole AND N. L. Zhang, A simple approach to Bayesian network computations, Proceedings of 10th Canadian Conference on Artificial Intelligence, pp. 171-178.

[11] G. COOPER, The computational complexity of probabilistic inference using bayesian belief networks, Artificial Intelligence, Vol. 42, Mar. 1990, pp. 405, 393.

[12] J. M. AGosta, Conditional inter-causally independent node distributions, a property of noisy-or models, Proceedings of the 7th Conference (1991) on Uncertainty in Artificial Intelligence, Los Angeles, California, United States: Morgan Kaufmann Publishers Inc., 1991, pp. 9-16.

[13] J. A. Rosen AND W. L. SMITH, Influence Net Modeling with Causal Strengths: An Evolutionary Approach, Command Control Research Technology Sumposium, Monterary, CA: 1996, pp. 699-708.

[14] K. C. Chang, P. E. Lehner, A. H. Levis, A. K. ZAIDI AND X. ZHAO, On Causal Influence Logic, George Mason University, 1994.

[15] F. DIEZ, Parameter adjustment in Bayes networks: The generalized noisy or-gate, Proceedings of the 9th Conference on Uncertainty in Artificial Intelligence, 1993, pp. 99-105.

[16] J. LEMMER AND D. GossinK, Recursive noisy ORa rule for estimating complex probabilistic interactions, IEEE Transactions on Systems, Man, and Cybernetics, Part B: Cybernetics, Vol. 34, 2004, pp. 2261, 2252.
[17] S. NAdKarni ANd P. P. Shenoy, A Bayesian network approach to making inferences in causal maps, European Journal of Operational Research, Vol. 128, Feb. 2001, pp. 479-498.

[18] Ş. Ö. ŞAhIN, F. ÜLEngIN AND B. Ülengin, A Bayesian causal map for inflation analysis: The case of Turkey, European Journal of Operational Research, Vol. 175, Dec. 2006, pp. 1268-1284.

[19] M. J. DRUZDZEL AND M. HENRION, Efficient Reasoning in Qualitative Probabilistic Networks, Proceedings of the 11th National Conference on Artificial Intelligence, 1993, pp. 548-553.

[20] L. W. WAGENHALS, I. SHIN AND A. H. LeVIS, Creating executable models of influence nets with colored Petri nets, International Journal on Software Tools for Technology Transfer (STTT), Vol. 2, Dec. 1998, pp. 168-181.

[21] S. HAIDER AND A. H. LEVIS, Modeling time-varying uncertain situations using Dynamic Influence Nets, Int. J. Approx. Reasoning, Vol. 49, 2008, pp. 488502.

[22] S. HAIDER, From dynamic influence nets to dynamic Bayesian networks: A transformation algorithm, Int. J. Intell. Syst., Vol. 24, 2009, pp. 919-933.

[23] LeE W. Wagenhals, J. T. Ried, R. J. Smille And ALEXANDER H. LEVIS, Course of Action Analysis for Coalition Operations, Proceedings of the 6th International Command and Control Research and Technology Symposium, Annapolis, MD: 2001.

[24] LeE W. Wagenhals AND AleXander H. LeVIS, Course of Action Analysis in a Cultural Landscape Using Influence Nets, Proceedings of the IEEE Symposium on Computational Intelligence for Security and Defense Applications, Honolulu: 2007.

[25] L. WENTZ AND L. WAGENHALS, Effects Based Operations for Transnational Terrorist Organizations: Assessing Alternative Courses of Action to Mitigate Terrorist Threats, Proceedings of the 9th International Command and Control Research and Technology Symposium, San Diego, CA: 2004.

[26] L. Wagenhals, R. JANSSEn AND E. DeGREGORIO, Model Interoperation for Effects Based Planning, Proceedings of the 9th International Command and Control Research and Technology Symposium, San Diego, CA: 2004.

[27] L. Wagenhals AND L. WENTZ, New Effects-based Operations Models in War Games, Proceedings of the 8th International Command and Control Research and Technology Symposium, Washington, DC: 2003.

[28] S. HAIDER, A. K. ZAIDI AND A. H. LEVIS, Identification of best sets of actions in Influence Nets, Int. J. Hybrid Intell. Syst., Vol. 5, 2008, pp. 19-29. 
[29] S. HAider, A. ZAidi AND A. Levis, A heuristic approach for best sets of actions determination in influence nets, Information Reuse and Integration, 2004. IRI 2004. Proceedings of the 2004 IEEE International Conference, 2004, pp. 600-605.

Received: August, 2011 Revised: June, 2012 Accepted: June, 2012

Contact addresses:

Sajjad Haider

Faculty of Computer Science Institute of Business Administration Karachi, Pakistan

e-mail: sahaider@iba.edu.pk

Muhammad Shafqat UNESCO

Islamabad, Pakistan e-mail: muhamad.shafqat@gmail.com

Shabih Haider

Department of Economics and Finance Institute of Business Administration

Karachi, Pakistan e-mail: shaider@iba.edu.pk
SAJJAD HAIDER received the B. Sc. (Hons.) degree in Statistics and M. Sc. degree in Computer Science from the University of Karachi, Pakistan, in 1997 and 1999, respectively, and the MS degree in Information Systems and the $\mathrm{Ph}$. D. degree in Information Technology from George Mason University, Fairfax, VA, in 2002 and 2005, respectively. He is currently an Associate Professor of Computer Science and the Head of the Artificial Intelligence Lab, Faculty of Computer Science, Institute of Business Administration, Karachi. He also holds a dual position of Honorary Associate at the University of Technology, Sydney where he is a member of the Innovation and Enterprise Research Lab. From 2005 to 2007, he worked as a Predictive Analyst at Fannie Mae, USA. His areas of interest include decision sciences, probabilistic reasoning, cognitive robotics, machine learning, computational intelligence and systems engineering.

Dr. Haider was a recipient of the 2004 Gary F. Wheatley Best Student Award at the Command and Control Research and Technology Symposium and the Best Paper Award from the International Council of Systems Engineering. He also won the best student awards during his B. Sc. (Hons.), M. Sc. and MS studies. He was recently awarded the Best Teacher Award by the Higher Education Commission of Pakistan.

MUHAMMAD SHAFQAT has done Bachelors in Business Administration (BBA) from the Institute of Business Administration, Karachi. He has worked as policy consultant at the Planning Commission of Pakistan devising the 'Innovation Roadmap' for Pakistan's 10th five-year development plan (Framework for Economic Growth 2012-2016). Since 2011 he has been working with UNESCO Islamabad, first as Foresight Consultant for its Science Sector, and now as Country Program Developer for the organization. His research interests are in the areas of crowdsourcing, social innovation and policy forecasting.

SHABIH HAIDER received the BE degree in Electronics from NED University and Masters of Business Administration degree from Institute of Business Administration, Karachi in 1979 and 1991, respectively. $\mathrm{He}$ is currently working as an Assistant Professor of Economics and Finance in the Institute of Business Administration, Karachi (IBA). He also serves as Management Advisor in Biogenics Pakistan Private Ltd. Prior to joining IBA, he taught at Hamdard University and worked as marketing manager in Alcatel, senior engineer in NESPAK (the largest engineering consultants in Pakistan) and senior application and service engineer in Zelin Private Limited. Mr. Haider's interests lie in cost accounting, financial economics and business statistics. 